\title{
Probiotic Interventions to Optimize the Infant and Child Microbiota
}

Received $30^{\text {th }}$ September

2017,

Accepted $29^{\text {th }}$ November 2017

Link to DOI:

10.25220/WNJ/V01.i2.0005

Journal Website:

www.worldnutrijournal.org

\author{
Yvan Vandenplas, $^{1}$ Koen Huysentruyt ${ }^{1}$
}

Department of Pediatrics, UZ Brussel, Vrije Universiteit Brussel, Brussels, Belgium

\begin{abstract}
The optimal healthy microbiota during early life still needs further evaluation. Pre- and probiotics are commonly used as supplementation in infant formula. Prebiotic oligosaccharides stimulate the growth of bifidobacteria aiming to mimic the gastrointestinal microbiota of breastfed infants. In general, results with prebiotics in therapeutic indications are disappointing. Studies suggest that probiotic supplementation may be beneficial in prevention and management of disease such as e.g., reducing the risk of necrotizing enterocolitis in preterm infants, prevention and treatment of acute gastroenteritis in infants, etc. Although many studies show promising beneficial effects, the longterm health benefits and eventual risks of probiotic supplementation during early life are not clear. It is likely that ongoing research will result in the use of specific probiotic organisms and/or prebiotic oligosaccharides during the first 1,000 days of life, with the goal to develop a healthy microbiota from conception over birth into the first two years of life with a lowered risk of infections and inflammatory events.

Keywords gastrointestinal microbiota, infant feeding, prebiotic, probiotic
\end{abstract}

\section{When is infant microbiota optimal?}

The question arises immediately: do we know the optimal healthy gastro-intestinal (GI) microbiota for the infant and child? The answer is probably negative. The important differences between the GI microbiota development between infants born through cesarean section versus natural delivery or standard infant formula feeding versus breastfeeding are well known. We also know that the GI microbiota of the mother is influenced by medications (antibiotics, anti-acid medications, etc), diet, stress and many other factors. ${ }^{1}$ The GI

\begin{tabular}{l}
\hline Corresponding author: \\
Yvan Vandenplas, $\quad$ Departmenten of \\
Paedaitrics, UZ Brussel, Laarbeeklaan 101, \\
1090 Brussels, Belgium.Tel: +3224775780; \\
e-mail: yvan.vandenplas@uzbrussel.be \\
\hline
\end{tabular}

microbiota of the breastfed baby born vaginally is in general considered as the "healthy microbiota", but depends on the GI microbiota of the mother. More data need to be collected to better define the "optimal healthy GI microbiota". The stepwise microbial gut colonization process may be initiated already prenatally by a distinct microbiota in the placenta and amniotic fluid. ${ }^{2}$ The clinical meaning of these findings needs to be further evaluated. A necrotizing enterocolitis (NEC) associated gut microbiota has been identified in meconium samples. Clostridium perfringens continues to be associated with NEC from the first meconium until just before NEC onset. In contrast, in postmeconium, increased numbers of staphylococci were negatively associated with NEC. ${ }^{3}$ Pre-term birth, cesarean section, formula feeding, antibiotic use and malnutrition have been linked to dysbiosis, which in turn is associated with several pathologies 
such as NEC, inflammatory bowel diseases, colic, and allergies.

Probiotics are living microorganism that, when administered in sufficient amount, have a health benefit for the host. Prebiotics are nondigestible food ingredients that beneficially affect the host by selectively stimulating the growth and/or activity of one or a limited number of bacteria in the colon, which can improve host health. Synbiotics are a mixture of pre- and probiotics.

Based on the current literature, a case can be made for the use of specific sets of probiotic organisms with the goal of promoting a healthy pregnancy to birth, and a healthy start to life with lowered risk of infections and inflammatory events. $^{4}$ The mechanisms of specific probiotic strains administered during the perinatal period suggest that probiotic interventions in early life can be envisaged for disease prevention in both healthy offspring and offspring at risk of chronic disease. There is evidence that manipulation of the infant microbiota by using pre- or probiotics can restore the ecological balance of the microbiota and may mitigate potential negative effects on the developing immune system, when use of antibiotics cannot be avoided. ${ }^{5}$

\section{Probiotics}

Unfortunately, the term "probiotic" seems to cause confusion. The taxonomic ranking for classification of probiotics is as follows: there is a domain (bacteria, fungi), a lineage (eg Furmicutes), a class (eg Lactobacilli), an order (eg Lactobacillales), a family, a genus, a species and subspecies. Most probiotics are bacteria, but the yeast Saccharomyces boulardii is one of the best studied, especially in gastroenterologic indications (Fig 1). Probiotics do not colonize the host. This means, that one to two weeks after stopping the administration of a probiotic strain, it disappears from the GI microbiota. The European Food Safety Authority considers "probiotics" as a medical claim, what results in the prohibition of the term on products or for communication by companies. More and more patents are given to specific probiotics with specific claims: fat metabolism, obesity, oral health, anticancer treatments, cardiovascular

disorders,

diabetes, immunomodulation, allergy, viral diseases such as AIDS, and others. A lot of research on probiotics is now focusing on other areas such as veterinary medicine and dermatology.

Traditional cuisines do contain a lot of fermented foods and thus probiotics. Fermented foods are foods produced or preserved by the action of microorganisms. The use of bacteria such as Lactobacillus results in the making of foods such as yoghurt and sauerkraut. The science of fermentation is known as zymology. Many pickled or soured foods are fermented as part of the pickling or souring process.

The market is overloaded with food supplements containing probiotics. However, there is no guarantee for the quality of such a product. There can be problems at the manufacturing level, but also at the level of survival during shelf life, or poor resistance to gastric acid. ${ }^{6-8}$ In other words, there is a world of difference between using probiotics as a healthy eating habit or using probiotics as medication.

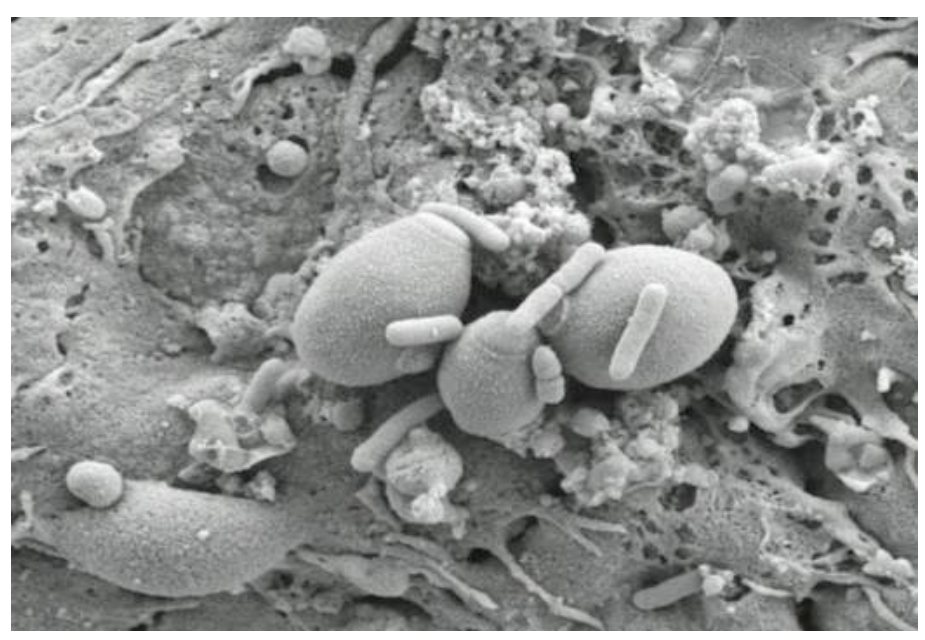

Figure 1 Illustration of the size difference between a yeast probiotic (Saccharomyces boulardii) and bacteria (Salmonella)

Probiotics can just be added to food, such as in infant formula. Probiotics are also added to foods such as chocolate and ice cream. Many yoghurts are enriched with probiotics. However, it is obvious that the targeted effect of probiotics in food differs in both situations substantially: while in infant formula there is a clear medical purpose, probiotics added to food such as ice cream and 
chocolate have a merely marketing goal claiming to improve general wellbeing. Probiotics used for medical purposes are often commercialized as pills or sachets. Some products are also on the market in liquid form and are administered as drops. Slow release tablets (e.g. to be put in a specially designed infant pacifier) were developed. ${ }^{9}$ Especially for respiratory tract indications, probiotics sprays have been developed. ${ }^{10}$ A novel probiotic delivery system has been developed in which probiotics are grown as a biofilm on microspheres, allowing enhanced efficacy with only a single treatment. ${ }^{11}$

It is beyond doubt that for specific medical purposes the effect of probiotic is strain-specific. This is illustrated by the following findings. Breastfeeding women received probiotic milk or placebo from 36 weeks of gestation up to 3 months postnatally while breast-feeding. ${ }^{12}$ The probiotic milk contained Lactobacillus (L.) rhamnosus GG, L. acidophilus La-5, and Bifidobacterium animalis subsp. lactis Bb-12. Only the L. rhamnosus GG bacteria colonized the children at 10 days and at 3 months of age. ${ }^{12}$ Different probiotic bacteria seem to have different ability to transfer from the mother to the child. This is in line with the finding that $\mathrm{L}$. rhamnosus strains present in breastmilk were isolated and characterized and should be further characterized. ${ }^{13}$ However, the relevance of strain specificity depends on the probiotic property that is looked for. Lactobacilli that survive gastric acid and bile will be fermented in the colon. Depending on the effect that is looked for, strain specificity is more or less important. The impact of probiotics on the development of the immune system in formula fed infants was recently illustrated by the findings of the TEDDY study. ${ }^{14}$ Early probiotic supplementation (at the age of 0-27 days) was associated with a decreased risk of islet autoimmunity when compared with probiotic supplementation after 27 days or no probiotic supplementation. $^{14}$ It was concluded that early probiotic supplementation may reduce the risk of islet autoimmunity in children at the highest genetic risk of diabetes. ${ }^{14}$

Many probiotic products are a combination of different strains. However, multi-species can as well do better than worse as single strains products. $^{15,16}$ In general, high dosages are more effective than low, although the number of dose- efficacy studies is limited. ${ }^{17}$ Although, some metaanalyses come exactly to the opposite conclusions: in patients with irritable bowel syndrome, single probiotics at a low dose and with a short treatment duration appear to be more effective in improving overall symptom response and quality of life. ${ }^{18}$ Older literature also suggest that the earlier probiotics are started (in the treatment of acute gastroenteritis), the greater the effect. ${ }^{19}$

In general, probiotics have the label to be "GRAS" (generally regarded as safe). However, side effects, mostly sepsis, are reported. But, probiotics have also been used safely in patients with immune deficiencies, showing a beneficial effect on growth only in HIV-positive infants. ${ }^{20}$ The increased use of probiotics in vulnerable immune insufficient preterm infants may lead to an increased risk of severe complications such as sepsis with the probiotic. $^{21}$

Although generalization should be avoided, as efficacy for medical indications is strain specific, it can be said that there is evidence that some strains do prevent atopic dermatitis (especially if also given to the mother during pregnancy). To date, expert bodies do not generally recommend probiotics for allergy prevention, although the World Allergy Organization (WAO) in their recently developed guidelines suggests considering using probiotics in pregnant women, during breastfeeding and/or to the infant if at high risk of developing allergic disease (based on heredity). ${ }^{22}$ However, in concordance with other expert bodies, the WAO guideline panel stressed the low level of evidence and the need for adequately powered randomized controlled trials and a more standardized approach before clinical recommendations on specific strains, dosages and timing can be given. ${ }^{22}$ Several meta-analyses have reported a moderate benefit of probiotics for eczema prevention, and the most consistent effect has been observed with a combined perinatal intervention in infants at high risk of allergic disease due to familial predisposition. ${ }^{22}$ Multistrain probiotics appeared to be most effective for eczema prevention. ${ }^{22}$

Probiotic strains have also been shown to prevent infectious disease such as community 
acquired and nosocomial gastroenteritis, respiratory tract and urinary tract infections. Infants receiving Bifidobacterium BB-12 were reported to have experienced fewer respiratory tract infections (risk ratio (RR): $0.87 ; 95 \%$ confidence interval (CI): $0.76,1.00 ; p=0.033)$ than controls. ${ }^{23}$ No significant differences between the groups were observed in reported GI symptoms, otitis media, or fever. ${ }^{23}$

Probiotics also decrease the incidence and severity of NEC in preterms. Strategies such as antenatal glucocorticoids, postnatal breast milk feeding, and cautious approach to enteral feeding failed to eliminate NEC because these strategies did not address the complexity of the pathogenesis. $^{24}$ Probiotics seem to be the most significant advance in NEC prevention at present because of the significant range of beneficial effects at various levels of gut function and defense mechanism. ${ }^{24}$ However not all studies are positive, as shown by a study with B breve BBG-001 for prevention of NEC and late-onset sepsis in very preterm infants. ${ }^{25} \mathrm{New}$ ways of administration of probiotics are developed, specifically for preterm infants. ${ }^{26}$ A single dose of Lactobacillus biofilm grown on biocompatible microspheres was shown to significantly reduce NEC incidence and severity in rats. ${ }^{26}$ Therefore, the risk/benefit should be considered before starting routine administration. Standard care in the Neonatal Intensive Care Units in the western world has resulted in a very low incidence of NEC. Oral probiotic given to VLBW infants do not affect neuromotor, neurosensory and cognitive outcomes at 18-24 months' corrected age. ${ }^{27}$ Rapid changes of nasogastric tubes may lead to a decreased incidence of NEC without the risk for sepsis. ${ }^{28}$

Probiotics also shorten the duration of infectious diseases, such as gastroenteritis. Probiotics added to infant formula have been shown in many older studies to possibly protect for infectious gastroenteritis, although some studies did not show a benefit. However, there was no study that suggested an increased risk of gastroenteritis. They are also effective in the prevention and treatment of antibiotic associated diarrhea (AAD). Moderate quality evidence suggests a protective effect of probiotics in preventing AAD (RR 0.46; $95 \%$ CI 0.35 to 0.61 ), with a number needed to treat of $10 .^{29}$
Probiotics may also have a role in infantile colic and in irritable bowel syndrome, especially older children and adults. The management of infantile colic in formula fed infants is still a challenge. L. reuteri DSM 17938 was ineffective in formula fed infants. ${ }^{30} \mathrm{~L}$. GG in infants treated in tandem with behavioral support and a cow's milk elimination diet did not provide additional treatment effect for diary-verified colic crying although parental report of crying suggested the probiotic intervention effective. ${ }^{31}$ A synbiotic (a mixture of seven probiotic strains plus FOS (Fructooligosaccharide)) significantly improved colic symptoms in comparison with placebo. ${ }^{32}$ Several studies were performed with L. reuteri DSM 17938 in breastfed infants presenting with infantile colic. ${ }^{33}$

Probiotics may also decrease regurgitation. Treated infants demonstrated a reduction in daily regurgitations at the end of treatment, three neonates in the placebo group only needed simethicone for GI pain, sIgA level was similar in both groups. $^{34}$

Probiotics result in a $10 \%$ eradication rate of Helicobacter pylori infection, although it is not known whether this is due to a true better eradication or better compliance to the eradication treatment because of a decrease of adverse effects (antibiotic associated diarrhea). Probiotics have been shown to be effective in the treatment of constipation in adults, but not in children. Literature on the efficacy of probiotics in inflammatory bowel disease is in general disappointing, certainly in pediatrics. The above list is not exhaustive, but indicates the broad spectrum of possible indications, going from general wellbeing over immune mediated diseases to infections. But negative results have also (seldom) been reported. Lactobacillus acidophilus (LAVRIA1) was reported to increase the risk of atopic dermatitis compared to placebo. ${ }^{35}$

\section{Probiotics during pregnancy}

Probiotics administered during pregnancy (and breastfeeding) to the mother can be found in the GI microbiota of the woman and has been shown for some strains to possibly have a preventive effect of the frequency and severity of atopic dermatitis in the infant. However, the literature on the effect of 
probiotics on the infant when given to the mother during pregnancy is quiet contradictory. According to some authors, currently evidence does not indicate that probiotic supplementation reduces the risk of developing allergy in children.

Current analysis of the role of probiotics in the prevention of atopic dermatitis reveals that a positive effect may be related to the type of probiotic strain used, the method of administration, onset time, as well as the dose size and duration of treatment. ${ }^{36}$ Panduru et al. ${ }^{37}$ concluded in a metaanalysis that probiotics have a protective role in atopic dermatitis prevention if they are administered during the pre- and postnatal period, in both the general and at allergic risk population. Maternal probiotic ingestion alone may be sufficient for long-term reduction in the cumulative incidence of atopic dermatitis, but not other allergy related diseases. ${ }^{38}$ The guideline of the World Allergy Organisation (WAO) panel determined that there is a likely net benefit from using probiotics resulting primarily from prevention of eczema. The WAO guideline panel suggests: a) using probiotics in pregnant women at high risk for having an allergic child; b) using probiotics in women who breastfeed infants at high risk of developing allergy; and c) using probiotics in infants at high risk of developing allergy. All recommendations are conditional and supported by very low quality evidence. $^{39}$

\section{Prebiotics}

Many of the production and conservation difficulties such as survival during production and shelflife with probiotics are not valid for prebiotics. Prebiotics stimulate the GI microbiota of the host, whereas probiotics just add one or couple of strains. According to some data, they may have prolonged effect. Some data suggest that if infant are supplemented from birth up to six months, that the bifidogenic effect can still be observed at the age of 12 months. ${ }^{40}$ Also prebiotics are generic, and findings of one prebiotic cannot be extrapolated to another one. ${ }^{41}$ The number of comparative and dose-efficacy studies is extremely limited.

The advantages of prebiotics in infants and children are mostly limited to prevention studies with infant formula supplemented with prebiotics oligosaccharides. While some studies show a benefit, others fail to do so, but the outcome in the prebiotic group is never worse than the comparator group. Most studies on the efficacy of prebiotics in therapeutic indications are disappointing. The effect of some prebiotic oligosaccharides on the gastro-intestinal microbiota is clear, as the effect on stool frequency and composition in non-constipated infants, bringing defecation pattern in formula fed infants closer to the pattern in breastfed infants. Literature is not conclusive on other possible effects such as decrease of infection, decrease of atopic dermatitis, and so on. Compared to probiotics, for which side effects are scarce but reported, adverse effects of prebiotics in infants are not reported.

\section{Synbiotics}

Data on the combination of pre- and probiotics in infants and children are quiet limited. Most studies regard short-term therapeutic interventions. Mean \pm standard deviation infection rates in infants followed up to 12 months were $4.9 \pm 3.2$ per infant per year in the B.lactis+GOS (Galactooligosaccharide)/FOS group and $4.5 \pm 3.0$ per infant per year in the B lactis group $(\mathrm{p}=0.18)$. Mean daily weight gain was slightly lower in the B.lactis + GOS/FOS than the B lactis group $(16.1 \pm 2.9$ vs $16.6 \pm 2.6 \mathrm{~g} /$ day, $\mathrm{p}=0.046)$, but was not clinically significant. ${ }^{42}$ Other outcomes were not significantly different between groups. Formulas containing B.lactis + GOS/FOS did not reduce infection rates beyond those containing only B lactis. ${ }^{42}$ Chang et al. ${ }^{43}$ conclude in their metaanalysis that evidence supports the use of synbiotics for the treatment of atopic dermatitis, particularly synbiotics with mixed strains of bacteria and for children aged 1 year or older. Nevertheless, infant formula companies promote the combination of pre- and probiotics in infant formula. Although this evolution does not seem to induce any increased risk for adverse effects, the benefit has not been shown.

\section{Conclusion}

The knowledge on the importance of the GI microbiota to the development of wellbeing and 
general health is increasing, and has been a focus of research during the past 10 years. The development of a healthy GI microbiota from conception throughout the first years of life will have lifetime long consequences. The use of prebiotics, probiotics and synbiotics in the prevention and treatment of different health conditions is increasing. If probiotic and prebiotic products are intended to be used in medical indications with the intention to claim a health benefit, at least two independent clinical studies are needed with the commercialized product. If products are commercialized with the intention to contribute to healthy eating habits, such high quality research is not needed. However, in that case, it is not justified to have any claim. In order to reduce confusion, a different terminology for "healthy foods with preand probiotics" and "specific products claiming a targeted health benefit" would be welcomed. The earlier proposed term "biotherapeutic agent" does not fulfill the requirements, as the concerned effects regard also prevention, probably even more than therapy.

\section{Open Access}

This article is distributed under the terms of the Creative Commons Attribution 4.0 International License (http://creativecommons.org/licenses/by/4.0/), which permits unrestricted use, distribution, and reproduction in any medium, provided you give appropriate credit to the original author(s) and the source, provide a link to the Creative Commons license, and indicate if changes were made.

\section{References}

1. Odamaki T, Kato K, Sugahara H, Hashikura N, Takahashi S, Xiao JZ, et al. Age-related changes in gut microbiota composition from newborn to centenarian: a cross-sectional study. BMC Microbiol 2016;16:90.

2. Collado MC, Rautava S, Aakko J, Isolauri E, Salminen S. Human gut colonisation may be initiated in utero by distinct microbial communities in the placenta and amniotic fluid. Sci Rep 2016;6:23129.

3. Heida FH, Zoonen, A . G. van, Hulscher JB, Bj TK, Wessels R, Kooi EM, et al. A necrotizing enterocolitis-associated gut microbiota is present in the meconium: results of a prospective study. Clin Infect Dis 2016;62:863-70.

4. Reid G, Kumar H, Khan AI, Rautava S, Tobin J, Salminen S. The case in favour of probiotics before, during and after pregnancy: insights from the first 1,500 days. Benef Microbes 2016;7:353-62.

5. Rutten NB, Rijkers GT, Meijssen CB, Crijns CE, Oudshoorn JH. K. van der, et al. Intestinal microbiota composition after antibiotic treatment in early life: the INCA study. BMC Pediatr 2015;15:204.

6. Hamilton-Miller JM, Shah S, Smith CT. Probiotic" remedies are not what they seem. BMJ 1996;312:55-6.

7. Vanhee LM, Goemé F, Nelis HJ, Coenye T. Quality control of fifteen probiotic products containing Saccharomyces boulardii. J Appl Microbiol 2010;109:1745-52.

8. Huys G, Botteldoorn N, Delvigne F. De , Heyndrickx M, Pot B, et al. Microbial characterization of probiotics--advisory report of the Working Group "8651 Probiotics" of the Belgian Superior Health Council (SHC. Mol Nutr Food Res 2013;57:1479-504.

9. Taipale $\mathrm{T}$, Pienihäkkinen $\mathrm{K}$, Isolauri $\mathrm{E}$, Jokela JT, Söderling EM. Bifido animalis subsp. lactis BB-12 in reducing the risk of infections in infancy. $\mathrm{Br} \mathrm{J}$ Nutr 2011;105:409-16.

10. Santagati M, Scillato M, Muscaridola N, Metoldo V. La , Stefani S. Colonization, safety, and tolerability study of the Streptococcus salivarius 24SMBc nasal spray for its application in upper respiratory tract infections. Eur J Clin Microbiol Infect Dis 2015;34:2075-80.

11. Olson JK, Rager TM, Navarro JB, Mashburn-Warren L, Goodman SD, Besner GE. Harvesting the benefits of biofilms: A novel probiotic delivery system for the prevention of necrotizing enterocolitis. Journal of pediatric surgery 2016;51(6):936-41.

12. Dotterud CK, Avershina E, Sekelja M, Simpson MR, Rudi K, Storrø O, et al. Does 
13. maternal perinatal probiotic supplementation alter the intestinal microbiota of mother and child. J Pediatr Gastroenterol Nutr 2015;61:200-7.

14. Panya M, Lulitanond V, Rattanachaikunsopon P, Srivoramas T, Chaiwong T. Isolation, Identification, and Evaluation of Novel Probiotic Strains Isolated from Feces of Breast-Fed Infants. Journal of the Medical Association of Thailand= Chotmaihet thangphaet 2016;99.

15. Uusitalo U, Liu X, Yang J, Aronsson CA, Hummel S, Butterworth $M$, et al. Association of Early Exposure of Probiotics and Islet Autoimmunity in the TEDDY Study. JAMA Pediatr 2016;170:20-8.

16. Grandy G Medina M, Soria R, Terán CG, Araya $\mathrm{M}$. Probiotics in the treatment of acute rotavirus diarrhoea. A randomized, double-blind, controlled trial using two different probiotic preparations in Bolivian children. BMC Infect Dis 2010;10:253.

17. Chapman CM, Gibson GR, Rowland I. In vitro evaluation of single- and multi-strain probiotics: Inter-species inhibition between probiotic strains, and inhibition of pathogens. Anaerobe 2012;18:405-13.

18. Shornikova AV, Casas IA, Isolauri E, Mykkänen H, Vesikari T. Shornikova AV. Lactobacillus reuteri as a therapeutic agent in acute diarrhea in young children. J Pediatr Gastroenterol Nutr 1997;16:1103-7.

19. Zhang Y, Li L, Guo C, Mu D, Feng B, Zuo $X$, et al. Effects of probiotic type, dose and treatment duration on irritable bowel syndrome diagnosed by Rome III criteria: a meta-analysis. BMC Gastroenterol 2016;16:62.

20. Rosenfeldt V, Michaelsen KF, Jakobsen M, Larsen CN, MØller PL, Pedersen P, et al. Effect of probiotic Lactobacillus strains in young children hospitalized with acute diarrhea. The Pediatric infectious disease journal 2002;21(5):411-6.

21. Steenhout PG, Rochat F, Hager C. The effect of Bifido lactis on the growth of infants: a pooled analysis of randomized controlled trials. Ann Nutr Metab 2009;55:334-40.
22. Zbinden A, Zbinden R, Berger C, Arlettaz $\mathrm{R}$. Case series of Bifidobacterium longum bacteremia in three preterm infants on probiotic therapy. Neonatology 2015; 107:56-9.

23. West CE. Probiotics for allergy prevention. Benef Microbes 2016;7:171-9.

24. Taipale TJ, Pienihäkkinen K, Jokela JT, Söderling EM. Bifidobacterium animalis subsp. lactis BB-12 in reducing the risk of infections in early childhood. Pediatr Res 2016;79:65-9.

25. Chen AC, Chung MY, Chang JH, Lin HC. Pathogenesis implication for necrotizing enterocolitis prevention in preterm verylow-birth-weight infants. J Pediatr Gastroenterol Nutr 2014;58:7-11.

26. Costeloe K, Hardy P, Juszczak E, Wilks M, Millar MR. P.I.S.C. (Probiotics). Bifidobacterium breve BBG-001 in very preterm infants: a randomised controlled phase 3 trial. Lancet 2016;387:649-60.

27. Olson JK, Rager TM, Navarro JB, Mashburn-Warren L, Goodman SD, Besner GE. Harvesting the benefits of biofilms: A novel probiotic delivery system for the prevention of necrotizing enterocolitis. Journal of pediatric surgery 2016;51(6):936-41.

28. Akar M, Eras Z, Oncel MY, Arayici S, Guzoglu N, Canpolat FE, et al. Impact of oral probiotics on neurodevelopmental outcomes in preterm infants. The Journal of Maternal-Fetal \& Neonatal Medicine 2017;30(4):411-5.

29. Petersen SM, Greisen G, Krogfelt KA. Nasogastric feeding tubes from a neonatal department yield high concentrations of potentially pathogenic bacteria [mdash] even $1 \mathrm{~d}$ after insertion. Pediatric research 2016;80(3):395-400.

30. Goldenberg JZ, Lytvyn L, Steurich J, Parkin P, Mahant S, Johnston BC. Probiotics for the prevention of pediatric antibiotic-associated diarrhea. Cochrane Database Syst Rev 2015;12.

31. Pärtty A, Lehtonen L, Kalliomäki M, Salminen S, Isolauri E. Probiotic Lactobacillus rhamnosus GG therapy and microbiological programming in infantile 
colic: a randomized, controlled trial. Pediatr Res 2015;78:470-5.

32. Kianifar H, Ahanchian H, Grover Z, Jafari S, Noorbakhsh Z, Khakshour A, et al. Synbiotic in the management of infantile colic: a randomised controlled trial. $\mathrm{J}$ Paediatr Child Health 2014;50:801-5.

33. T1 H, Matsuyama M, David M, Hill RJ. Infant colic - What works: a systematic review of interventions for breast-fed infants. J Pediatr Gastroenterol Nutr 2016;62:668-86.

34. Garofoli F, Civardi E, Indrio F, Mazzucchelli I, Angelini M, Tinelli C, et al. The early administration of Lactobacillus reuteri DSM 17938 controls regurgitation episodes in full-term breastfed infants. Int $\mathbf{J}$ Food Sci Nutr 2014;65:646-8.

35. Taylor AL, Dunstan JA, Prescott SL. Probiotic supplementation for the first 6 months of life fails to reduce the risk of atopic dermatitis and increases the risk of allergen sensitization in high-risk children: a randomized controlled trial. J All Clin Immunol 2007;119:184-91.

36. Rather IA, Bajpai VK, Kumar S, Lim J, Paek WK, Park YH. Probiotics and atopic dermatitis: an overview. Front Microbiol 2016;7:507.

37. Panduru M, Panduru NM, Sălăvăstru CM, Tiplica GS. Probiotics and primary prevention of atopic dermatitis: a metaanalysis of randomized controlled studies. J Eur Acad Dermatol Venereol 2015;29:23242.

38. Simpson MR, Dotterud CK, Storrø O, Johnsen R, Øien T. Perinatal probiotic supplementation in the prevention of allergy related disease: 6 year follow up of a randomised controlled trial. BMC Dermatol 2015; $15: 13$.

39. Fiocchi A, Pawankar R, Cuello-Garcia C, Ahn K, Al-Hammadi S, Agarwal A, et al. World Allergy Organization-McMaster University Guidelines for Allergic Disease Prevention (GLAD-P): Probiotics. World Allergy Organ J 2015;8:4.

40. Salvini FJ. Riva E, Salvatici E, Boehm G, Jelinek J, Banderali G, Giovannini M. A specific prebiotic mixture added to starting infant formula has long-lasting bifidogenic effects. Nutr 2011;141:1335-9.

41. Veereman-Wauters G, Staelens S. Van de, Plaskie K, Wesling F, Roger LC, et al. Physiological and bifidogenic effects of prebiotic supplements in infant formulae. $\mathrm{J}$ Pediatr Gastroenterol Nutr 2011;52:763-71.

42. Bocquet A, Lachambre E, Kempf C, Beck L. Effect of infant and follow-on formulas containing B lactis and galacto- and fructooligosaccharides on infection in healthy term infants. J Pediatr Gastroenterol Nutr 2013;57:180-7.

43. Chang YS, Trivedi MK, Jha A, Lin YF, Dimaano L, García-Romero MT. Synbiotics for Prevention and Treatment of Atopic Dermatitis: A Meta-analysis of Randomized Clinical Trials. JAMA Pediatr 2016;170:236-42. 